\title{
Une tradition disputée : enjeux identitaires dans une communauté des Andes boliviennes
}

\section{Vanina Bouté}

\section{(2) OpenEdition}

\section{Journals}

Édition électronique

URL : https://journals.openedition.org/jsa/1523

DOI : 10.4000/jsa.1523

ISSN : 1957-7842

\section{Éditeur}

Société des américanistes

\section{Édition imprimée}

Date de publication : 5 juin 2003

Pagination : 125-148

ISSN : 0037-9174

\section{Référence électronique}

Vanina Bouté, "Une tradition disputée : enjeux identitaires dans une communauté des Andes

boliviennes », Journal de la Société des américanistes [En ligne], 89-2 | 2003, mis en ligne le 05 juin 2008, consulté le 02 septembre 2022. URL : http://journals.openedition.org/jsa/1523 ; DOl : https://doi.org/ $10.4000 /$ jsa. 1523 


\title{
UNE TRADITION DISPUTÉE : ENJEUX IDENTITAIRES DANS UNE COMMUNAUTÉ DES ANDES BOLIVIENNES
}

\author{
Vanina BOUTÉ *
}

\begin{abstract}
Cet article se propose d'analyser les conflits qui opposent les différents groupes sociaux d'une communauté de l'Altiplano bolivien ; ces conflits résultent notamment de la déliquescence des anciens liens de pouvoir établis avant la Réforme agraire. C'est principalement la vision du passé - à perpétuer, à réinventer ou à rejeter - qui est au cœur de ces conflits. Nous verrons ainsi comment ces visions plurielles du passé, objet de tensions et de renégociations entre les groupes, s'expriment principalement à travers des célébrations religieuses. [MотS cLÉs : Bolivie, traditionnalisme, indigénisme, identité, conflits, marka, changement social.]
\end{abstract}

La tradición reñida : diversidad identitaria en una comunidad de los Andes bolivianos. Este artículo se da a la tarea de analizar los conflictos que enfrentan a los diferentes grupos sociales de una comunidad del Altiplano boliviano. Dichos conflictos tienen su origen en la desintegración paulatina de las antiguas relaciones de poder establecidas antes de la Reforma agraria de 1953. En las distintas concepciones del pasado-que se perpetúa, se recrea o se rechaza - radica la razón misma de estos conflictos. Examineramos de qué manera las múltiples concepciones del pasado, que son motivos de tensiones y renegociaciones entre los grupos, se expresan principalmente en las distintivas celebraciones religiosas. [PALABras CLAves : Bolivia, tradicionalismo, indigenismo, identidad, conflictos, marka, cambios sociales.]

The keenly contested tradition : identity issues in a comunity of the bolivian Andes. This paper aims at analysing conflicts between various social groups within a community of the Bolivian Altiplano; those conflicts can primarily be traced back to the decay of traditional power bonds that existed before the Agrarian Reform of 1953. Underlying these conflicts is a representation of the past, to be perpetuated, reinvented or rejected. We will thus see how these various representations, creating tensions and renegociations between groups, are mainly expressed through differentiated religious celebrations. [KeY worDS : Bolivia, traditionalism, indigenism, identity, conflicts, marka, social changes.]

Comment les différentes composantes d'une société locale andine fortement hiérarchisée réagissent-elles lorsque le principe qui sous-tendait l'ordre social se

* Doctorante en ethnologie, Laboratoire d'ethnologie et de sociologie comparative, UMR 7535, Maison de l'archéologie et de l'ethnologie, Université Paris X-Nanterre [vaninab@hotmail.com].

Journal de la Société des Américanistes, 2003, 89-2, pp. 125-148. O Société des Américanistes. 
trouve soudainement balayé, laissant un vide d'autorité ? San Andrés, communauté locale des environs de La Paz, reposait sur une structure sociale hiérarchique et coercitive, dominée par de grands propriétaires fonciers, descendants d'Espagnols et de Métis. La Réforme agraire de 1953 allait bouleverser cet ordre social, rejetant l'ancienne autorité.

Une rupture s'est progressivement mise en place entre chacune des composantes de cette communauté andine, qui s'est traduite par le développement de discours et de pratiques distinctifs visant à l'élaboration d'une identité propre, définie hors des liens qui unissaient auparavant les groupes. De nombreux éléments du passé n'en continuant pas moins de peser fortement, ces derniers ont réagi en tentant soit de se les approprier ou les remodeler, soit au contraire de les abandonner ostensiblement et leur substituer des valeurs nouvelles. Mon propos, dans cet article, est de rendre compte de l'attitude des trois principaux groupes sociaux composant San Andrés face à ce qui est pensé comme traditionnel, attitude qui trouve essentiellement - mais pas seulement - une expression religieuse. C'est donc au rôle idéologique ${ }^{1}$ de la tradition, de ses usages ou de son refus, dans l'élaboration de l'identité distinctive des groupes sociaux, que sont consacrés les développements qui suivent.

\section{LA MARKA SAN ANDRÉS : UNE CONFIGURATION BOULEVERSÉE}

A $120 \mathrm{~km}$ de $\mathrm{La} \mathrm{Paz}^{2}$, sur les hauts plateaux boliviens, San Andrés de Machaqa est une marka (appelée également canton dans certains documents officiels) peuplée par quelque 2000 familles $^{3}$. Le terme marka, d'origine aymara, désigne une forme préhispanique d'organisation territoriale regroupant des communautés dispersées sur un vaste espace. Les marka ont connu d'importantes mutations : avec l'arrivée des Espagnols notamment, elles furent démembrées et recomposées en de plus petites unités territoriales qui conservèrent cependant la même appellation. Ces nouvelles marka étaient désormais dotées d'un centre villageois, ancien bourg de réduction ${ }^{4}$. Au centre de la marka San Andrés fut ainsi construit, au XVII ${ }^{\mathrm{e}}$ siècle, le village du même nom. Son agencement spatial est à peu près celui des autres villages fondés par les Espagnols : une place carrée au centre, où se trouvent l'église et les principaux bâtiments administratifs, avec des rues à angle droit découpant des pâtés de maisons.

Sans entrer plus en détail sur les périodes coloniale et républicaine ${ }^{5}$, je donnerai ici un aperçu de l'organisation sociale et territoriale de la marka San Andrés juste avant 1953 et de la façon dont les vecinos et les comunarios en ont conservé le souvenir. Vecinos signifie littéralement « habitants du bourg » et, par extension, ce terme peut désigner une catégorie de notables ${ }^{6}$. Comunarios a pour sens littéral "habitants des communautés» (ou des ayllu ${ }^{7}$ ). La marka San Andrés est aujourd'hui divisée en six ayllu ${ }^{8}$ répartis autour du village central. Les 
ayllu sont des unités sociales et géographiques dont les habitants disent avoir des ancêtres mythiques communs, les achachilas ${ }^{9}$, premiers fondateurs du territoire. Chaque ay/lu est divisé en plusieurs estancias, lieux de résidence et de production de familles étendues et apparentées entre elles. Les activités d'élevage et d'agriculture ${ }^{10}$ sont organisées, au sein de chaque estancia, par un chef de groupe. La propriété de la terre est généralement pro-indivise, c'est-à-dire que les terres d'une même famille ne se morcellent pas en propriété privée pour ses membres.

Dans le village de San Andrés vivaient jusqu'en 1953 des vecinos, affirmant généralement être les descendants d'Espagnols et de Métis, des obreros (artisans) et quelques comunarios. Installés sur la place du village, les vecinos détenaient le pouvoir administratif et les principales charges d'autorité de la zone. Dans leur majorité, ils étaient commerçants, voire artisans. Ils s'étaient appropriés les terres environnantes qu'ils louaient aux paysans aymaras, les comunarios, ou les leur faisaient exploiter. Il n'y eut cependant pas d'expansion de grandes haciendas, comme cela fut le cas dans les marka voisines. Les vecinos considéraient les comunarios comme appartenant à la « race indienne ", elle-même perçue comme arriérée, paresseuse, superstitieuse, sale et consommant coca et alcool ${ }^{11}$. Ils distinguaient les « Indiens des communautés » des « Indiens du village », les premiers étant largement plus dévalorisés que les seconds. Parmi ces derniers, les obreros étaient des propriétaires fonciers plus ou moins aisés, métis ou comunarios souvent identifiés, hors du village et dans les villes, comme cholos $^{12}$ et possédant une maison dans les rues attenantes à la place. Exemptés des prestations dues à l'administration et aux notables, certains d'entre eux employaient même, à l'instar des vecinos, des comunarios. Enfin, quelques rares comunarios, dont l'estancia jouxtait les terres du village, étaient spécialement affectés au service des vecinos et de la paroisse et pouvaient posséder une maison à la périphérie du village.

Les relations des vecinos avec les autres habitants de la marka étaient globalement fondées sur des rapports de domination, mélange de clientélisme et de paternalisme. Les commuarios, en raison du joug que faisaient peser sur eux les vecinos, ne portaient pas ces derniers dans leur cœur. Malmenés, exploités comme domestiques, par le maire et le curé, ils étaient également obligés de fournir des travaux pour le village des vecinos. Un comunario raconte : «Entre gens de San Andrés, nous n'avons jamais été égaux. Lorsque j'étais petit, les vecinos voulaient diriger, organiser les autres et, de ce fait, certains nous maltraitaient. C'était une vieille habitude de ces gens-là de maltraiter les gens pauvres et humbles. C'était comme cela ».

Le village central de San Andrés était le cœur administratif et cérémoniel de la marka et, à ce titre, les comunarios se devaient de participer aux constructions, aux réparations des bâtiments publics et aux fètes religieuses, occupant un espace qui leur était attribué sur la place ${ }^{13}$. C'étaient surtout les fêtes religieuses qui se déroulaient à San Andrés, qu'elles fussent collectives ou consacrées à un $a y l l u$ spécifique. Elles permettaient aux membres des ayllu d'affirmer leur apparte- 
nance commune à la marka et leurs liens avec le village, « espace commun fondamental des membres des ayllu » (Rivière 1983, p. 47).

En 1952, le pouvoir politique en Bolivie passa aux mains du Movimiento nacionalista revolucionario (MNR) qui, en opposition au vieil ordre oligarchique, instaura de profondes transformations dont la Réforme agraire de 1953. Les changements engendrés par cette Réforme jouèrent considérablement sur l'organisation de la marka. Les grands propriétaires terriens, ou vecinos, furent dépouillés de leurs privilèges; les terres furent redistribuées aux habitants des communautés, lesquelles accédèrent à un statut juridique indépendant. La Réforme agraire provoqua notamment la prolifération de nouveaux bourgs et de foires (ferias) propres à chaque $a y l l u$ qui, en décentralisant les activités économiques et politiques, amoindrirent le rôle du village. Dans certains cas (et ce fut celui de San Andrés), ces nouvelles créations (ainsi que d'autres comme la multiplication des écoles rurales et la formation des syndicats paysans) renforcèrent également le processus de fragmentation entre les communautés. L'ancienne unité organisée autour du bourg - centre économique, politique et religieux perdit progressivement tout sens : les vecinos partirent s'installer dans les grands centres urbains du pays, tandis que les comunarios, dégagés des services et corvées, se recentrèrent autour de leur ayllu, qu'ils dotèrent d'un petit hameau faisant office de village central. Dans le même temps, le bourg de San Andrés fut peu à peu investi par les obreros et les domestiques des vecinos qui avaient pu faire l'acquisition d'une maison et, souvent, d'une tienda (épicerie, parfois buvette) et qui s'approprièrent les principales charges publiques. Progressivement, ces familles se coupèrent de leur ayllu d'origine, rejetant l'agriculture et l'élevage, pratiques qu'elles considéraient comme socialement dégradantes. Reprenant à leur compte un élément de la relation qui prévalait avant 1953 entre vecinos et comunarios, certains de ces nouveaux villageois donnèrent à leur tour leurs terres en métayage à d'autres comunarios, souvent des membres de leur parenté ${ }^{14}$. Enfin, le processus d'autonomie des ayllu par rapport au village s'est encore accentué en 1994 avec la promulgation de la Loi de Participation Populaire, qui accorde la personnalité juridique aux communautés paysannes ou indigènes et décentralise la politique des travaux publics jusque-là située dans le village central. Si, dans d'autres régions de l'Altiplano, cette loi a rattaché de fait les communautés à d'anciens bourgs, à San Andrés, les ayllu qui étaient déjà dotés de leurs propres bourgs ont acquis une indépendance administrative par rapport au village. Comme l'a noté Lavaud (1998), ces nouvelles bourgades entrent aujourd'hui en compétition avec les anciens villages coloniaux et les plus importants d'entre elles acquièrent progressivement des caractéristiques semblables à celles des villages traditionnels.

Au sein de la marka San Andrés contemporaine, on peut schématiquement ${ }^{15}$ identifier trois principaux groupes (dont les membres se différencient souvent en plusieurs sous-catégories) : 
- les vecinos qui, malgré leur résidence urbaine (à La Paz généralement), continuent de se nommer entre eux vecinos et sont considérés comme tels par les habitants de la marka. Ils reviennent massivement au village à l'occasion de la fête de la Sainte Patronne, la Virgen del Rosario ;

- les comunarios, paysans aymaras, vivant dans les ayllu ;

- enfin, ceux que nous appellerons, par commodité, le groupe des «nouveaux villageois " ${ }^{16}$; il s'agit d'une vingtaine de familles résidant actuellement dans le village et aspirant à occuper la position locale d'autorité des anciens vecinos. Ces " nouveaux villageois » tentent de se démarquer des comunarios (par l'usage de l'espagnol, par le port de vêtements plus urbains, etc.) et les regardent dédaigneusement comme des paysans hostiles au progrès et se confortant dans leur misère, reprenant par là les discours jusqu'alors tenus par les vecinos. Par ailleurs, ils cherchent à reproduire à leur profit l'ancienne relation de domination entre vecinos et comunarios.

Avec la disparition de la structure sociale coercitive, comment se fait à présent le partage du territoire entre ces groupes ? Comment, dans cette nouvelle configuration, chaque groupe exprime-t-il l'affirmation d'une appartenance à la marka ? À la suite des changements introduits par la Réforme agraire, on a observé une décomposition des liens entre groupes, ces liens d'interdépendance (n'excluant pas tensions et conflits) qui faisaient la spécificité de la marka comme unité sociale. La crise économique qui, depuis les dix dernières années, a entraîné les vecinos urbanisés à revenir vers le monde rural pour tenter d'y recouvrer leur autorité a ensuite contribué à accentuer les tensions qui se sont cristallisées autour du groupe des nouveaux villageois. En voulant reproduire l'ancien ordre social, ces derniers heurtent à la fois les intérêts des vecinos et ceux des comunarios. Les vecinos voient d'un mauvais œil ces «intrus » sur leur territoire: " comme nous, nous voulons être citadins, ces gens-là veulent être vecinos ", reconnaissent-ils. Mais ils leur dénient une quelconque identité " vecinale » ${ }^{17}$ et, surtout, toute possibilité de l'acquérir. Car les vecinos aiment à se caractériser par l'éducation et l'instruction; ils soulignent à l'envi l'ignorance des nouveaux villageois : « Ils n'ont pas cette éducation que n'importe qui peut avoir. Ce sont des gens pauvres et médisants ». De leur côté, les comunarios, affranchis du joug des vecinos depuis la Réforme agraire, n'entendent pas accepter l'autorité que les nouveaux villageois tentent de leur imposer. Les rumeurs, propageant l'image du vecino autoritaire et violent, sont prêtes à renaître lorsque des conflits apparaissent entre les comunarios et les nouveaux villageois. Un comunario raconte : « Les vecinos autrefois nous détestaient et nous maltraitaient. C'était ainsi autrefois. Maintenant, les vecinos sont partis et les gens du village viennent tous sans exception du campo (monde rural). Mais il y a, comme on dit, des germes du passé dans ce village. Ceux du village viennent des communautés mais ils détestent ceux des communautés et, nous aussi, nous les détestons ». Le village et ses nouveaux habitants sont perçus comme une menace potentielle pour les 
communautés : l'affranchissement des services obligatoires aux vecinos qu'a permis la Réforme agraire ne semble pas encore un gage suffisant d'indépendance pour pallier la menace d'une ingérence des nouveaux villageois dans la vie des communautés.

Finalement, chacun des trois groupes - vecinos, nouveaux villageois, comunarios - tend aujourd'hui à construire son identité en rupture et opposition avec les autres groupes, et cela autour de la notion de tradition. Afin de comprendre et d'illustrer ce processus, on va examiner les discours et les pratiques des membres de ces groupes, en montrant que les tensions autour de ce qui est conçu comme traditionnel s'expriment principalement par des pratiques religieuses différenciées.

\section{LES VECINOS : L'APPROPRIATION D'UNE TRADITION}

Avec la crise économique qui affecte les centres urbains, et notamment La Paz, le milieu rural que les vecinos ont quitté acquiert de nouveau un certain prestige à leurs yeux. La ville est de moins en moins en mesure de répondre à leurs attentes, d'autant qu'ils n'ont pas su ou pu retrouver dans la société urbaine le statut dominant qu'ils avaient dans leur milieu d'origine. Depuis le début des années 1990, les aspirations des vecinos se sont donc progressivement retournées vers le monde rural et le village. Des familles de vecinos y reviennent de plus en plus souvent, et pas seulement pour des célébrations religieuses. Le fils d'un vecino remarquait : " mon père est revenu faire semer sur un bout de terrain que nous avions conservé. Cela fait bien vingt ans qu'il n'avait pas fait semer ». Car certains vecinos espèrent recréer en milieu rural un pôle économique qui puisse apporter des ressources complémentaires aux revenus obtenus en ville ; ils ont ainsi fondé le Centro Acción dont l'idée phare est la modernisation et l'urbanisation du village ${ }^{18}$. D'autres reconnaissent leur engouement, une certaine nostalgie pour la maison familiale qu'il faut restaurer : «Ainsi, nous allons pouvoir revenir plus souvent ». Et, de fait, les vecinos entendent bien reconquérir leur place, occupée par les nouveaux villageois. Cette reconquête s'exprime notamment par l'exaltation d'un terroir et d'une tradition qu'ils disent être " andine » et la leur propre, à l'exclusion de tout autre groupe de la marka.

\section{Le traditionalisme "vecinal 》 : retour au terroir et folklorisation}

Les occasions de rappeler les liens avec la terre et la nature, les us et coutumes du campo, sont nombreuses dans la vie quotidienne des vecinos. À travers leurs paroles, il apparaît en effet que la vie au village est parée de toutes les qualités : l'air y est pur, la santé ne peut être qu'excellente, les aliments sont naturels, rien n'y est coûteux (ce n'est pas comme en ville où tout se paie), la tranquillité est bienfaisante (pas comme en ville où tout est bruyant et stressant), il n'y a pas de 
voleurs, pas de délinquance. Et, surtout, le campo est riche de ressources naturelles qu'il faudrait pouvoir exploiter. Certains vecinos affirment même que ceux qui disent que l'Altiplano est pauvre sont mal informés ou paresseux. Tous imaginent des projets pour l'exploitation du campo : élevage intensif, agriculture, ventes en ville, etc. Ce réancrage des vecinos au campo est nourri par une appropriation de ce qu'ils conçoivent comme une culture indienne prestigieuse. Ces discours sur les supposées valeurs du monde rural et indien sont présents non seulement chez les vecinos mais aussi chez de nombreux Aymaras qui ont quitté le monde rural pour devenir des citadins. Ces Aymaras ont transformé le mépris dont ils sont l'objet en tant qu'Indiens en élément positif dans la représentation d'eux-mêmes qu'ils construisent pour eux et pour les autres ${ }^{19}$.

De fait, les aspects que vecinos et migrants indiens retiennent sont ceux mis en valeur par un certain folklore national, utilisé notamment pour la promotion du tourisme mais qui participe malgré tout à la construction d'une identité nationale. Les aspects au travers desquels ce folklore est exprimé sont multiples et il serait diffīile d'en donner ici même un aperçu ${ }^{20}$. Rappelons brièvement que cette promotion d'un folklore national fut originellement lancée par les mouvements indigénistes ${ }^{21}$, à travers essentiellement les groupes de danse urbains, dont les parades (lors du Carnaval d'Oruro ou de la Fiesta del Gran Poder à La Paz) font figure aujourd'hui d'événements nationaux. Les éléments que les vecinos retiennent sont, entre autres, une image pittoresque de l'Indien, porteur de costumes aux couleurs chatoyantes, aux mœurs exotiques, descendant du prestigieux empire inca dont il aurait su conserver les coutumes supposées ancestrales. Les vecinos font un amalgame entre les Aymaras et les Incas, cette confusion étant peut-être due à certains mouvements indianistes ${ }^{22}$. Certaines ONG ont également un impact sur les représentations que se forgent les vecinos du monde rural et, particulièrement, de l'Indien. Les activités de ces ONG (la plus importante, le CIPCA ou Centro de investigación y de promoción del campesino andino, étant installée dans la marka voisine de Jesús de Machaqa) sont tournées vers le développement rural. Depuis une vingtaine d'années, ces ONG ont mis en place des outils de promotion de la culture aymara : radios locales, diffusion de films ${ }^{23}$ et organisation de festivals dits « aymaras », etc. Il est intéressant de noter que la plupart de ces actions sont imprégnées d'une idéologie indianiste et d'une certaine conception de ce qu'est la tradition aymara, dont les vecinos s'inspirent largement. Paradoxalement, cette promotion d'une culture andine avait été entreprise, avant tout, afin de revaloriser l'image que les Aymaras avaient de leur propre culture, dépréciée autrefois par les vecinos. Ces derniers mettent ainsi en avant la connaissance qu'ils pensent avoir de cette culture afin de montrer qu'ils sont, en dépit de leur résidence urbaine, liés à un univers terrien - la marka-, dont ils comprennent et respectent parfaitement l'esprit.

Les fêtes religieuses - principalement celle de la sainte patronne du village, la Virgen del Rosario - sont les moments où les vecinos s'attachent le plus ostensi- 
blement à reproduire ces « traditions indiennes », qu'ils qualifient fréquemment de tradiciones ancestrales. Ils mettent alors en avant les aspects les plus exotiques de cette indianité retrouvée. Les rituels à la Pachamama (qui, dans la cosmologie andine, correspond à la divinité de la terre) et autres références possibles à une tradition aymara y tiennent une place importante. Par exemple, lorsqu'il s'agit de tuer les animaux pour le repas des invités, le patron de la fête ou pasante ${ }^{24}$ à laquelle j'ai assisté - un vecino - effectue un rite de propitiation à la Pachamama en lui offrant les cours frémissants des animaux. Il dispose le cariño $^{25}$, un tissu coloré utilisé par les femmes aymaras comme châle ou pour le portage de boissons alcoolisées et de feuilles de coca. Les personnes chargées de découper les bêtes sont toujours des comunarios : aujourd'hui, ces derniers sont payés pour effectuer cet acte, mais cela ne manque pas d'évoquer les anciens services qu'ils devaient rendre au vecino propriétaire de leurs terres. Pendant le découpage, le vecino, patron de la fête, ne cesse d'observer la façon dont ils l'exécutent en les morigénant : "Faites bien attention à ceci, à cela, c'est notre culture, il faut la respecter ». Ces mêmes comunarios me feront, par la suite, part de leur étonnement : le passé n'est pas si loin où les vecinos raillaient les « superstitions absurdes » des Aymaras, superstitions dans lesquelles ils rangeaient les cultes à la Pachamama.

Les vecinos se présentent donc comme les dépositaires d'un savoir indien et insistent sur le respect de valeurs et d'habitudes célébrées par eux comme typiquement andines. Il en est ainsi de l'ayni : celui-ci, propre aux communautés andines, consiste ordinairement en un échange réciproque de services de même nature entre deux personnes, créant des liens durables entre les membres d'une communauté. Aujourd'hui, l'ayni n'est que rarement effectué par les habitants du village et par les comunarios qui ont recours à la mink'a - échange de services qui peuvent être de nature différente - ou au travail salarié. L'ayni, tel que les vecinos l'effectuent, ne concerne jamais directement un travail à accomplir mais plutôt des échanges de biens lors de fêtes : on offre à un vecino d'une marka voisine un feu d'artifice, du bétail ; celui-ci rendra la pareille lorsque le vecino donateur en aura besoin. En revanche, lorsque les vecinos s'adressent à des comunarios pour effectuer certains travaux (le plus souvent la réparation de leur maison au village), ils leur donnent un salaire comme le font également les nouveaux villageois. Plus qu'à une nécessité pratique, le recours à l'ayni semble répondre à leur désir d'adoption d'une valeur andine, connotée positivement et utilisée en des occasions exceptionnelles.

Certaines habitudes culinaires sont également mises en avant et définies comme emblématiques de la tradition indienne aymara. La consommation de viande de lama est depuis peu - suite au mouvement lancé par des ONG étrangères pour en promouvoir la commercialisation -fort prisée par les vecinos (qui la consommaient pourtant auparavant). L'un d'eux raconte : "Les Aymaras ont honte de manger de la viande de lama et, pourtant, c'est leur nourriture ances- 
trale. Nous, nous n'avons pas honte. Au contraire, nous disons : c'est plein de vitamines, il faut en manger ! ». Pour la majorité des paysans boliviens, le lama est considéré comme la viande du pauvre, par opposition au bœuf servi dans les restaurants de la capitale. Mais, pour les vecinos, le respect et le bon accomplissement de cette supposée tradition aymara sont les signes de leur éducation et de leur intelligence. Et le fait que les comunarios et les villageois ne les suivent pas sur ce point leur apparaît comme une preuve supplémentaire de leur stupidité et, implicitement, de leur infériorité ${ }^{26}$.

Cette recréation de l'image de l'Indien par les vecinos rend encore plus vif leur rejet des comunarios aymaras et, plus directement encore, des nouveaux villageois. Comunarios et nouveaux villageois sont désormais considérés non plus comme les dépositaires d'une culture andine, ni comme des « Indiens », mais comme de simples paysans, le terme paysan (campesino) étant devenu, depuis la Réforme agraire, un substitut de l'ancienne appellation péjorative d'Indio. La ségrégation contre les Aymaras persiste : à présent, ce n'est plus l'Indien aymara qui est dévalorisé mais le paysan ainsi que sa prétendue absence de tradition. Cet accaparement d'une supposée tradition indienne par une élite blanche ou métisse est largement présent lors de célébrations urbaines. Par exemple, lors du Carnaval d'Oruro, une bourgeoisie urbaine aisée se travestit en Indiens sauvages ou démons précolombiens, mais les Aymaras sont totalement absents du défilé (Abercrombie 1992). Par leur mise en scène d'une certaine image de l'Indien, lors d'une cérémonie qui exclut les comunarios, les vecinos reproduisent finalement ce schéma en l'introduisant au sein du monde rural.

Cette nouvelle représentativité de l'Indien, conçue et idéalisée par les vecinos, n'a plus grand chose à voir avec la réalité des habitants de la marka. Cette culture est rattachée au passé par le biais d'un folklore et d'une muséographie fortement développée ${ }^{27}$. Elle permet, par le remodelage de l'image de l'Indien, d'exalter un passé national glorieux ${ }^{28}$. Ce phénomène n'est pas en soi nouveau si l'on se réfère aux mouvements indigénistes présents à Cuzco dès le début du siècle ; ce qui l'est davantage, c'est que ce phénomène n'est plus l'apanage d'une élite intellectuelle et blanche mais il est, désormais, le fait d'une autre frange de la population bolivienne, les vecinos déclassés.

Ces quelques exemples montrent comment la « fabrication de la tradition ${ }^{29}$ par les vecinos est produite à travers des discours et des pratiques et intégrée dans un contexte particulier, principalement celui des fêtes religieuses. À ces occasions, les vecinos tentent de compenser leur position de migrants (residentes ${ }^{30}$ ) - qui les met dans une situation moins valorisante que celle des nouveaux villageois -, grâce à l'affirmation d'une certaine autochtonie au sein du village. Jadis célébrations de l'appartenance commune de tous les groupes de la marka, les fêtes mettent aujourd'hui principalement en scène les vecinos qui, pour ce faire, tendent à monopoliser les charges religieuses locales. 


\section{Les charges religieuses : l'accaparement par les vecinos}

Les cultes rendus aux différents saints vénérés dans la marka donnent lieu à des fêtes religieuses importantes qui rythment l'année. Ces célébrations permettent à un groupe ou à la collectivité tout entière de maintenir sa propre intégrité et d'assurer sa reproduction : elles établissent, d'une part, des rapports de réciprocité entre le saint célébré et les membres du groupe concerné ; elles assurent, d'autre part, une concentration et une redistribution (à la fois symbolique et matérielle) de prestations et de biens entre les diverses unités domestiques de ce groupe. Autrefois, la célébration des fêtes était un outil important de consolidation des liens entre les ayllu et le village, leur centre cérémoniel. Mais les tensions qui se sont récemment développées entre vecinos citadins, nouveaux villageois et comunarios ont modifié cette configuration. Ces tensions sont particulièrement manifestes dans le fonctionnement actuel du système des charges religieuses.

Dans la marka, le terme « cargos » (« charges ») désigne des fonctions politiques ${ }^{31}$ ou religieuses spécialisées. Le système des charges constitue le cadre institutionnel et traditionnel de la collectivité. Idéalement, grâce aux charges, l'ensemble des membres d'une marka - que cela soit au niveau individuel, familial ou des estancias - est intégré dans un même système de solidarité et de hiérarchie. L'accès aux charges, politiques et religieuses, dépendait de la capacité d'un jeune ménage à mettre en valeur ses terres et à développer son activité économique de façon à pouvoir dégager un surplus de biens qui pouvait être redistribué lors de l'accomplissement des charges ; et le prestige provenait de la générosité dans la redistribution des biens. Actuellement, au village (mais aussi dans les communautés), ces charges ne permettent plus cette intégration : elles sont devenues des objets de discorde et de luttes; elles ne sont plus réparties entre tous. $\mathrm{Si}$, une dizaine d'années auparavant, des nouveaux villageois ou des comunarios des environs pouvaient recevoir une charge pour une célébration religieuse, le retour massif des vecinos au village a considérablement modifié cette donne. Comme le souligne Wachtel (1990, p. 146), « cette redistribution ramène constamment vers le bas de l'échelle la plupart des membres de la communauté, d'autre part elle maintient au sommet ceux qui, peu nombreux, s'y trouvaient déjà ».

La principale charge religieuse qui retiendra notre attention est celle de pasante. Le pasante occupe la fonction clé d'organisateur d'une des fêtes religieuses parmi celles qui scandent le calendrier rituel des ayllu ou de la marka entière : il jouit de tous les pouvoirs pour la fête dont il est responsable et prend en charge les coûts qu'elle occasionne ${ }^{32}$. Il patronne en quelque sorte la fête et, de ce fait, met en jeu sa crédibilité. La fonction de pasante implique donc - en sus d'un réseau de relations étendu - d'avoir un capital financier important. Or le départ des vecinos et de certains villageois vers les grands centres urbains du pays a accentué les différences sociales entre ces derniers et ceux restés dans la marka San Andrés. Lors de la fète de la Virgen del Rosario, les pasantes sont au nombre de 
cinq et l'on assiste à une surenchère entre les vecinos pour acquérir du prestige, lequel passe par des investissements considérables. Ce prestige est couronné par la remise de trophées aux meilleures comparsas (groupe de danseurs invités par le pasante ; chaque groupe exécute une danse précise, danse à laquelle est associé un costume spécifique que tous les danseurs doivent revêtir). Pour chaque comparsa, les danseurs sont au nombre de trente en moyenne, mais peuvent compter jusqu'à soixante personnes ; les fanfares (bandas) accompagnant les danseurs sont engagées à La Paz et composées de dix musiciens pour les plus modestes, de vingt à trente pour les plus riches. Les types de danses choisis par les pasantes sont également hiérarchisés : la Morenada est la danse la plus prestigieuse ; c'est également la plus onéreuse, au vu des costumes et du nombre de musiciens qu'elle nécessite. Elle est, selon Albó et Preiswerk (1986, p. 105), « l'expression de ceux qui ont pleinement réussi leur vie familiale et sociale ». La Caporal est également une danse prestigieuse. C'est toujours l'une ou l'autre de ces danses qui est choisie par les vecinos et qui remporte les trophées. En effet, si un nouveau villageois a la charge de pasante, il choisit une danse moins coûteuse comme la Oriental ou la Kullawada. La remise des trophées, par un jury composé des vecinos les plus aisés, est censée récompenser les danseurs les plus vifs, la fanfare la plus dynamique et la meilleure coordination ; de fait, elle semble se fonder avant tout sur la magnificence déployée par un pasante. L'observation des fêtes permet de mettre en relief les inégalités qui se forment entre les titulaires des charges et de mieux comprendre pourquoi les nouveaux villageois - et les comunarios qui le désireraient encore - peuvent difficilement participer à la fête del Rosario sans perdre la face car ils ne peuvent rivaliser avec les citadins dans le déploiement de luxe. En raison de liens de compérage (compadrazgo ${ }^{33}$ ) avec le vecino organisateur, des comunarios des estancias environnantes peuvent certes être conviés à la fête de la Virgen del Rosario et, même, intégrer le groupe de danseurs du pasante. Mais, depuis quelques années, la participation aux processions dansées, caractéristiques des fêtes religieuses boliviennes, est de plus en plus chère ${ }^{34}$.

Un des caractères fondamentaux de la charge est, selon Molinié (1982, p. 162), la réciprocité : « les prestations seront minutieusement rendues lorsque le collaborateur assumera lui-même la charge, suivant exactement les règles de l'ayni des travaux agricoles ». De fait, il est d'usage, le premier jour de la fête, que les invités offrent au pasante boissons alcoolisées et feuilles de coca. La nature et la quantité de ces dons permettent dans une certaine mesure de moduler les relations d'échange que les invités souhaitent établir avec le pasante, soit que les invités désirent de cette façon recevoir du pasante le même présent lorsqu'ils rempliront à leur tour une charge religieuse, soit qu'ils lui sont redevables d'un présent de même nature, lorsqu'ils étaient eux-mêmes pasantes. Un autre exemple de cette réciprocité peut être observé le dernier jour de la fête : les invités accrochent à la veste ou au chapeau du pasante et de sa femme une petite somme d'argent. Ce geste n'est pas considéré comme une obligation, néanmoins 
le montant de chaque somme ainsi que les noms des donateurs sont scrupuleusement notés par un assistant. Les donateurs, et uniquement eux, sont alors conviés le lendemain à de nouvelles réjouissances. Les comunarios et les nouveaux villageois qui ne peuvent assumer financièrement une charge de pasante ne peuvent pas non plus participer en tant qu'invités aux fêtes, se trouvant ainsi exclus des célébrations religieuses.

L'accomplissement d'une charge de pasante est lié au prestige et inversement : « celui qui n'est pas pasante une fois dans sa vie ne reçoit que le mépris de ceux d'ici » nous confie un vecino dont le fils devait recevoir la charge pour la fête de la Virgen del Rosario. La pression sociale pousse les individus à remplir la fonction de pasante d'une fête afin de pouvoir obtenir une reconnaissance au sein du groupe. Les vecinos, soucieux d'affirmer leur nouvel ancrage dans l'espace villageois, se montrent dès lors avides d'affirmer cette appartenance de façon éclatante, via la possession d'une charge. La distribution des charges ne s'étend plus à l'ensemble du groupe, mais se fait au sein d'un réseau de parenté et de relations ; dans l'ivresse de la fête, les successeurs, qui font partie de l'entourage du pasante, se présentent spontanément ${ }^{35}$. De sorte qu'il s'opère un accaparement des charges religieuses par une catégorie sociale aisée et urbanisée, composée exclusivement de vecinos et de certains migrants, originaires du village, bien intégrés dans le milieu urbain et qui tendent à occulter leur origine aymara. En vertu du principe de réciprocité, la distribution des charges se réalise dans un groupe de plus en plus restreint, souvent au sein de la parenté. Par ailleurs, la différenciation entre les charges religieuses se fait de plus en plus criante ; celles-ci sont considérées comme plus ou moins prestigieuses en fonction des danses choisies (et de leur coût), mais également en fonction de la fête à laquelle elles sont rattachées. La fête de la Virgen del Rosario, patronne du village, est la plus prestigieuse : c'est celle à laquelle tous les vecinos se rendent. Ils sont en revanche absents des autres célébrations (fête de San Andrés, Noël, Carnaval, etc.) et ce sont les nouveaux villageois qui en assument les charges, moins coûteuses ${ }^{36}$. Incapables de se mesurer à la formidable prodigalité du pasante et des autres invités, vecinos ou migrants, du coup exclus de la célébration religieuse la plus prestigieuse du village, les nouveaux villageois tentent à leur tour - avec succès - d'exclure de ces fêtes les comunarios des environs du village qui voudraient assumer une charge. De leur côté, les comunarios se sont, depuis une vingtaine d'années, progressivement désengagés de ces célébrations, refusant tout lien avec le village et ses habitants.

Loin de renoncer à s'affirmer comme membres légitimes du village ou de la marka San Andrés, nouveaux villageois et comumarios réagissent avec force contre cette mise à l'écart de la part des vecinos. Leurs stratégies diffèrent, mais ont néanmoins en commun d'opposer leur propre vision du passé à celle du groupe vis-à-vis duquel ils se distinguent : pour les nouveaux villageois, il s'agit donc des vecinos, pour les comunarios, ce sont les nouveaux villageois. 


\section{LES COMUNARIOS : LE CHOIX DE LA CONVERSION}

C'est essentiellement à partir des années 1950, parallèlement à la Réforme agraire, que des groupes religieux évangéliques se sont implantés solidement en Bolivie ${ }^{37}$. À San Andrés, ce mouvement a été favorisé par la proximité de la frontière avec le Chili, d'où provient l'Église évangélique pentecôtiste, ainsi que par l'implantation, sur la marka voisine de Santiago de Machaqa, d'une ONG dirigée par la Mission suédoise libre. Outre ces deux Églises, les Adventistes et les Témoins de Jéhovah se sont aussi installés dans la région. Les temples sont relativement neufs et entretenus avec le plus grand soin. Les règles à observer par les convertis sont multiples : interdiction de boire de l'alcool, de participer aux fêtes catholiques, de faire des sacrifices aux divinités autochtones ${ }^{38}$. Les tensions entre comunarios, d'une part, vecinos et nouveaux villageois, de l'autre, se sont traduites, depuis une vingtaine d'années, par un processus de conversion massive des comunarios à ces courants évangéliques. Les facteurs d'adhésion des commuarios sont sans doute nombreux et complexes. Nous ne prétendons pas en faire une étude approfondie mais nous voulons montrer ce en quoi cette adhésion peut être liée, d'une part, à la diffículté d'accéder aux charges, d'autre part, à un rejet de certains éléments associés au passé.

Les habitants des ayllu, n'ayant plus accès aux charges religieuses, refusent de se rendre au village lorsqu'ils y sont conviés et ne participent même plus à la « fête des ayllu " ${ }^{39}$. "Les comumarios venaient il y a trente ans au village. Maintenant non, car ils sont hermanos. Ils ne veulent plus rien dépenser pour les fêtes, ils ne veulent pas être pasante. Il y en a même qui deviennent hermanos avant la fin de la fête pour ne pas avoir à payer. Ici, nous sommes tous catholiques ", explique fièrement un nouveau villageois. Hermano ("frère ») est un terme utilisé par les adeptes des groupes évangéliques pour s'auto-désigner et, de fait, de nombreux comunarios eux-mêmes énoncent sans détour s'être convertis pour échapper aux coûts croissants qu'entraînent les charges religieuses "catholiques ». Remplir une charge religieuse est devenu tellement coûteux que, pour certains, cela peut signifier la ruine ou, en tout cas, l'absence de reconnaissance sociale qu'elle garantissait naguère. Ainsi, au moment des passations de charges, à la fin des fêtes, des drames éclatent souvent : certains déclinent la charge, quitte à perdre la face ; d'autres s'enfuient des lieux de la fête et du village vers les communautés ou la ville.

Entre exclusion des fêtes du village et marginalisation ou ruine au sein de leur propre communauté, les comunarios trouvent dans les nouvelles Églises une solution pour échapper aux pratiques des fêtes catholiques. Comme le souligne Rivière (1997, p. 90), « à la différence des charges traditionnelles, aucune richesse, aucun savoir particulier ne sont exigés pour entrer dans l'Église [pentecôtiste]. Dans les cultes, les fidèles retrouvent le droit de parler et d'être écoutés, facultés dont ils ont été privés dans "l'autre monde" où certains secteurs ont accaparé la 
parole et n'écoutent plus les "pauvres" lors des assemblées communales ». Les nouvelles Églises interdisent la participation aux rituels et au système des charges - exception faite des charges politiques - et exhortent à une vie tournée vers une certaine frugalité, de sorte que les comunarios peuvent éviter la ruine tout en conservant une certaine dignité. Mieux, ils renversent le processus d'exclusion dont ils faisaient l'objet par une attitude dynamique : refuser la célébration des fêtes catholiques et adopter de nouveaux cultes.

Ce phénomène de conversion des comunarios aux groupes évangéliques serait-il uniquement imputable à des raisons économiques ? Pour les comunarios, les fêtes célébrées au sein de l'Église catholique ne sont plus l'occasion d'afrirmer une appartenance commune à la marka; elles apparaissent en quelque sorte vidées de toute substance et rattachées à un passé négatif. "Ceux de ma communauté disent que les vecinos se sont bien mal conduits ; ils nous malmenaient avec des fouets. Les comunarios s'en souviennent encore et c'est pour cela qu'ils refusent de venir au village ", explique un comunario. Ainsi, la dissidence religieuse serait également pour les commarios le moyen de marquer leur opposition à la domination des vecinos et de leurs successeurs, les villageois, domination à laquelle le catholicisme est fortement associé dans leur esprit. L'un d'entre eux se souvient : "Avant, les habitants des six ayllu devaient se relayer pour venir travailler à l'église. Il y avait donc six personnes qui venaient, à tour de rôle, chaque semaine pour sonner les cloches, matin, midi et soir. Ainsi étaient les vecinos, des paresseux ». Le catholicisme est perçu à travers le prisme d'un passé lourd de soumission - les services obligatoirement rendus à la paroisse et l'image souvent négative du curé, blanc et abusif, donc du côté des vecinos ${ }^{40}$. À la suite d'événements obscurs ${ }^{41}$, le curé a dû définitivement quitter le village en 1986. Les nouveaux villageois racontent à ce propos: "Les comunarios ont renié le Père Pepe. Ils ont été ingrats avec lui. C'est pour cela que le Père s'en est allé ».

Les comunarios recherchent, par des pratiques religieuses autres que catholiques, un moyen de célébrer l'identité de leur groupe et son ancrage dans la marka. Il y a création d'un ordre différent de celui jusqu'alors observé ; les conversions massives dans les communautés agissent dans le sens de l'affirmation d'une nouvelle identité des comunarios. Le rejet du catholicisme et, surtout, l'adhésion à des groupes religieux qui se positionnent en deçà des nouvelles valeurs promues par l'Église catholique - culture andine et autochtonie folklorisée - correspondent à une volonté de se définir en opposition à la fois aux vecinos et aux nouveaux villageois. En se situant en dehors des codes et des rites de l'ancienne société, les comunarios n'échappent pas seulement à l'inflation vertigineuse du coût des charges : soustraits à une organisation politico-religieuse qui les plaçait au bas de la hiérarchie sociale, ils font échouer la tentative des nouveaux villageois qui voulaient imposer leur autorité à travers la réactualisation d'un type ancien de relation de domination ${ }^{42}$. 
Parallèlement aux avancées du protestantisme, la présence du clergé catholique se fait discrète et de plus en plus d'églises sont laissées à l'abandon. L'Église catholique met l'accent sur une "évangélisation depuis la culture » : les messes célébrées sont dites en aymara, la cosmogonie andine est reprise dans certains prêches, etc. À l'intérieur de la marka, s'opposent finalement deux pôles religieux et sociaux : le catholicisme qui s'appuie sur ce qui est conçu comme étant la tradition autochtone pour fonder sa propre légitimité, soutenu en cela par les nouveaux villageois, partisans du maintien du passé dans le sens où ils aspirent à occuper la position des anciens vecinos; les nouveaux groupes religieux, refusant toute forme traditionnelle et œuvrant pour une société nouvelle, soutenus par les comunarios $^{43}$.

TODOS SANTOS, FÊTE DES MORTS ET CULTE DES ANCÊTRES : LA LÉGITIMATION DES NOUVEAUX VILLAGEOIS

Les nouveaux villageois, face au rejet par les comunarios de leur prétention à la domination et face à leur mise à l'écart du système des principales charges religieuses, tentent d'exprimer leur identité de nouveaux vecinos par une célébration différente des fêtes traditionnelles. Depuis une dizaine d'années, ils se sont, par exemple, approprié l'ancienne fête de l'ayllu Qollana, fête dite « de San Andrés ». Les villageois sont actuellement les seuls à célébrer cette fête qu'ils disent être la leur et dont ils écartent soigneusement les comumarios. À l'instar des vecinos célébrant la fête de la sainte patronne du village et tenant à l'écart les autres groupes, les nouveaux villageois ont construit à leur tour leur propre célébration en se dotant d'un saint prestigieux à leurs yeux - puisqu'il donne son nom au village - et en confortant, par le biais de cette fête, leur appartenance au village.

Cette célébration de leur légitimité est encore plus manifeste lors de la fête de la Toussaint (Todos Santos). À cette occasion, les nouveaux villageois réaffiriment leur appartenance au village en s'inventant une autochtonie sur ce territoire qu'ils ont doté d'ancêtres aymaras, les achachilas. En effet, la cosmogonie chrétienne s'étant intégrée aux croyances anciennes des Aymaras, aux côtés des figures du panthéon chrétien prennent place la Pachamama, le soleil (Intitata), la lune (Paxsimama), l'esprit de la maison, les esprits de certains lieux, les âmes des enfants morts sans baptême, les âmes des morts du commun et une catégorie d'esprits appelés les achachilas. Ces derniers sont des intermédiaires entre les divinités et les hommes. On dit qu'ils résident à proximité des hommes, c'est-àdire dans les collines, parfois dans des croix ou des monolithes qui entourent le territoire habité. Molinié (1986, p. 157) note qu'une « de leurs fonctions était de marquer l'appropriation et de borner un territoire ». Certains achachilas sont définis comme les ancêtres, premiers occupants des lieux et fondateurs du groupe. 
À travers les cultes rendus aux achachilas, les Aymaras d'un ayllu réitèrent leur sentiment d'appartenance au territoire et les liens qui les unissent par ces ancêtres.

Jusqu'au départ des vecinos dans les années 1950, quatre autels recevaient les cultes aux achachilas. Monticules de terre surmontés d'une croix, cadran solaire, niche dans une petite tour, ces lieux de culte étaient érigés au sommet des quatre collines entourant le village. Il existait par ailleurs une correspondance entre chacun de ces lieux et les quatre coins de la place du bourg. Lors de la " fête des ayllu », les membres de chaque ayllu occupaient, sur la place du village, le lieu qui leur était réservé, y dansaient et terminaient la célébration par une libation à l'autel correspondant à cet emplacement. L'achachila honoré était défini comme leur ancêtre, titulaire donc de deux autels, l'un situé au sein de l'ayllu, l'autre dans le village. Par ce biais, étaient aflïrmées l'appartenance de tous à la marka et la cohésion des membres des ayllu. Avec le repli des comunarios et la rupture de leurs relations avec les occupants du village, ces lieux de culte ont été progressivement délaissés. Toutefois, depuis le retour des vecinos, les nouveaux villageois se les ont réappropriés ainsi que les divinités qui y siègent. Si quelques personnes reconnaissent que les achachilas ont toujours été présents autour du village, la plupart des nouveaux villageois affirme que ces achachilas étaient certes présents autrefois, mais méconnus de tous et qu'ils se seraient en quelque sorte " révélés » à eux après le départ des vecinos. Cette " révélation ", soulignent-ils, est le signe de leur élection par des instances supérieures comme habitants légitimes du village. Et si, avant, ces achachilas étaient définis comme les ancêtres des membres des ayllu, les nouveaux villageois expliquent qu'aujourd'hui ces achachilas sont leurs propres ancêtres, maîtres du village et de ses environs. Doter le village espagnol d'ancêtres aymaras est aussi une manière, pour eux, de confirmer leur antériorité sur les vecinos. Cette stratégie leur permet à la fois de se poser en véritables ordonnateurs de l'espace villageois, de s'imposer comme un groupe uni par des ancêtres communs et, dès lors, de s'opposer, en tant que groupe, aux vecinos et aux comunarios. Le culte principal aux achachilas se déroule d'ailleurs pendant la fête catholique de Todos Santos.

Ordinairement, lors des fêtes catholiques, le parcours des processions religieuses suit un tracé précis et significatif : le mode d'occupation de l'espace est une sorte de réaffirmation de l'ancrage du groupe sur son territoire et la fête construit l'identité du groupe autour du saint qu'il honore. Une comparaison entre deux types de parcours - l'un classique effectué par les vecinos lors de la fête de la Virgen del Rosario (sainte patronne du village) et l'autre récemment introduit et effectué par les nouveaux villageois, lors de Todos Santos - permet de mettre en lumière deux modes différents et concurrents d'appropriation de l'espace villageois.

Un des critères d'appartenance au village, mis en avant par les vecinos, est celui de leur filiation à des ancêtres espagnols, qu'ils disent être les fondateurs et les premiers occupants de l'espace villageois (il s'agit en fait d'une fiction, le 
village ayant originellement été créé afin de « réduire » les Aymaras dans un lieu circonscrit). Les maisons des familles fondatrices, les plus illustres, sont regroupées autour de la place centrale, face à l'église, cœur du village. Lors des célébrations religieuses où les vecinos sont présents, le parcours est particulièrement réalisé dans l'espace de la place du village. Celui-ci est divisé en quatre quartiers qui sont symboliquement représentés par les quatre coins de la place où les pasantes accomplissent, chacun leur tour, une libation en l'honneur du (ou de la) saint(e), sollicitant par ce geste sa bénédiction pour le territoire. L'espace célébré et ainsi marqué ne s'étend donc guère au-delà de la place, haut lieu de l'identité " vecinale », même si, par extension, les marges du village, c'est-à-dire les quartiers, sont rattachées à la célébration par les libations.

Les nouveaux villageois et les comunarios des estancias attenantes sont les seuls à célébrer Todos Santos au village, cette fête étant délaissée par les vecinos. La procession fait un parcours très différent de celui effectué au cours des autres fềtes. Le $1^{\text {er }}$ novembre, après les prières et les libations pour les morts au cimetière, les comunarios se dispersent, poursuivant les célébrations dans la cellule familiale, dans leur estancia. En revanche, pour les nouveaux villageois, commence un rituel tout à fait différent. Par petits groupes, dansant au son des flûtes en bois, ils entreprennent l'ascension des collines qui entourent le bourg et y sollicitent la protection des achachilas pour l'année à venir. Les lieux où passe la procession débordent largement l'espace de la place centrale puisque le cortège part du cimetière (en contrebas) et traverse la place pour se rendre vers les collines situées aux quatre points cardinaux du village. Tout se passe comme si ce parcours, instauré depuis la « révélation » des achachilas, était l'occasion de célébrer l'identité du groupe et de revendiquer son ancrage dans un territoire fondé par les ancêtres légendaires et divinisés. En superposant à un agencement de l'espace « vecinal» un espace proprement autochtone - car lieu des achachilas -, les nouveaux villageois paraissent insister, eux aussi, sur leur appartenance au village. Leur parcours encercle la place, l'inscrit dans un tracé plus large ; il leur permet, semble-t-il, de revendiquer un espace plus important où le territoire « vecinal » n'est plus qu'une partie et non un centre absolu. Inscrire leur légitimité dans l'espace villageois, ce serait enfin réaffirmmer la validité du rôle centralisateur du village et sa supériorité sur les ayllu.

Dans un essai sur les usages du passé dans les Andes péruviennes, Urton (1993, p. 108) remarque qu'au sein des collectivités sociales andines la «tradition » donne lieu à une pluralité d'élaborations concurrentes lorsque « il n'existe pas de force claire et hégémonique exerçant un contrôle dans le présent ou détenant le privilège incontestable de représenter la nature de cette société dans le passé ». Il en va de même dans la marka de San Andrés : la renégociation du passé par chacun des trois groupes sociaux - comunarios, nouveaux villageois et vecinos - donne naissance à des traditions reformulées, différentes, antagonistes et qui se manifestent tout particulièrement lors de célébrations religieuses. Celles-ci 
deviennent l'événement au cours duquel se manifestent les revendications identitaires et les tensions entre ces trois groupes, chacun cherchant à se repositionner par rapport aux nouveaux enjeux de pouvoir au sein de la marka.

Dans une société en pleine transformation, les nouveaux villageois se veulent les gardiens d'un ordre antérieur à 1953. Désireux d'acquérir le statut de vecinos, ils rêvent de perpétuer l'ancienne relation entre vecinos et comunarios et d'instaurer leur domination sur les habitants des ayllu. Toutefois, leur confrontation avec les vecinos les pousse à exprimer de façon nouvelle leur appartenance au village ; ils ont recours dès lors à des éléments - traditionnels dans les $a y l l u$ mais récemment introduits dans le village - afin de revendiquer une autochtonie sur le territoire qu'ils occupent. Cette attitude des nouveaux villageois se heurte aux conceptions des comunarios et des vecinos : les premiers rejetant toute velléité de domination des nouveaux villageois, les seconds s'inscrivant dans une nouvelle lecture du passé et refusant de leur concéder le rôle de nouveaux vecinos. Dans un village à reconquérir, les vecinos introduisent, par les fêtes, d'autres éléments, largement inspirés d'un folklore urbain et ancrés dans un passé lointain revisité par leurs soins. Cette refonte du passé leur permet d'aflirmer plus que jamais un fort attachement à ce qui est conçu comme un univers terrien, l'espace du village, et une tradition inca, réinventée, dont ils se veulent les dépositaires. Pour les comumarios, au passé, c'est-à-dire à la période antérieure à 1953, est rattaché le souvenir négatif de leur domination par les vecinos. La contestation s'élève donc contre un ordre dénoncé comme caduc ou aberrant et s'exprime par l'adoption de pratiques religieuses nouvelles, lesquelles, en rejetant en bloc l'ordre traditionnel, leur permettent de tourner le dos au village et à ses habitants.

Les tensions entre ces trois groupes se manifestent à travers des visions divergentes du passé : pour les comunarios et les villageois, le passé est associé aux vecinos; tandis que, pour ces derniers, la tradition est celle des Incas, dont les actuels Aymaras ne seraient que les héritiers ignorants. Dans le même temps, les affrontements pour la conquête du village passent, tant chez les vecinos que chez les nouveaux villageois, par des "emprunts » à un groupe méprisé, les comunarios ; lesquels ont depuis rejeté ces coutumes reprises par les autres. Les usages de la tradition, le traditionalisme ou, au contraire, son refus et l'adoption d'une certaine modernité ne semblent devoir être définis qu'à travers les conflits qui structurent les rapports entre les trois groupes. *

* Manuscrit reçu en mai 2003, accepté pour publication en novembre 2003 


\section{NOTES}

Remerciements : à Antoinette Molinié pour sa disponibilité et ses précieux conseils.

1. Je me réfère ici à l'idée d'Apter $(1965$, p. 83) de « tradition comme idéologie ». Cet auteur définit le traditionalisme comme ne résidant pas seulement « dans l'adoption de normes traditionnelles parce qu'elles semblent favoriser la solution des problèmes courants [... mais] plutôt dans l'appel à ces normes traditionnelles parce qu'elles sont anciennes et en raison de l'aflirmation de leur caractère sacré ou de leur supériorité idéologique ».

2. Plus exactement dans la province Ingavi. Celle-ci est divisée en quatre sections : Viacha, Waqui, Tihuanacu, Desaguadero. Le territoire de San Andrés de Machaqa appartient à la section de Viacha.

3. 1798 familles en 1988 (Diagnóstico socioeconómico 1988) et 2200 en 1998 (Distritación de la Provincia Ingavi, Secretario nacional de Participación Popular, trabajo borrador, 1996).

4. Lors de la colonisation, les Espagnols imposèrent le regroupement des populations autochtones, dont l'habitat était dispersé, dans des villages appelés pueblos de reducción et ce, afin de mettre un terme à la dispersion des indigènes, de permettre leur christianisation et de faciliter leur rassemblement pour le travail, la perception des tributs, et l'incursion des Européens et des Métis dans les terres indigènes.

5. Nous ne pouvons dans cet article retracer l'historique de San Andrés de Machaqa. En revanche, nous nous concentrerons sur une période qui apparaît comme centrale dans la détermination des changements sociaux actuels, soit les années précédant la Réforme agraire de 1953. Pour une histoire des périodes coloniales et républicaines à San Andrés, nous renvoyons le lecteur à l'ouvrage de Choque (1988). À propos du processus de fragmentation des marka durant ces périodes, voir Choque (1988) et Paredes (1955). D’un point de vue plus général, se reporter enfin aux articles de Bouysse-Cassagne, de Platt et de Harris, dans le numéro d'Annales consacré à l'Anthropologie historique des sociétés andines (1978, n5-6, septembre-décembre).

6. Selon Marie Helma (citée in Bourricaud 1962, p. 17), cette définition est héritée de l'époque coloniale ; il s'agissait de «l'habitant qui avait pu prouver au conseil de la ville que la maison qu'il habitait dans la ville lui appartenait en propre, qu'il était propriétaire d'armes ». Sur les vecinos, voir aussi la thèse de Cécile Claudel (2000).

7. Le terme de "communauté » peut recouvrir, dans la dénomination des espaces andins, des réalités différentes et désigner la marka, l'ayllu ou l'estancia. Nous avons choisi de conserver les dénominations utilisées par Roberto Choque (1988) et celles des habitants de San Andrés (les deux concordant) : le terme d'" ayllu» (ou "communautés ») désigne dorénavant les grands ensembles territoriaux qui composent la marka.

8. Ces six ayllu sont répartis en deux moitiés : celle " du haut » comprend les ayllu Qollana, Choque, Levita ; celle « du bas », les ayllu Alto Achacana, Bajo Achacana et Yaru.

9. En aymara, achachila est également un terme de parenté désignant le grand-père paternel ou maternel.

10. Les quelque 2000 familles de San Andrés vivent essentiellement de l'élevage de lamas et de moutons ainsi que de certaines cultures vivrières (pomme de terre, orge, quimua).

11. Cette opposition sociale se traduit dans les discours et les comportements des vecinos par la vision d'un monde concentrique dont le centre, le village " urbanisé » et "civilisé », est un espace connoté positivement et s'oppose à une périphérie « sauvage, désordonnée, dangereuse ", peuplée par les comunarios. Isbell (1978) et Abercrombie (1990), entre autres, ont relevé et analysé cette opposition.

12. Le terme " cholo » désigne « un secteur intermédiaire formé d'individus qui s'étaient progressivement séparés individuellement, et non en tant que groupe social, du groupe indien pour accepter une série de valeurs, essentiellement économiques, du groupe non indien " (Albó 1977).

13. D’après les témoignages que j'ai recueillis auprès des habitants du village, les membres de chaque ayllu, lors des fètes, se voyaient attribuer une rue, par laquelle ils accédaient en dansant à leur 
emplacement sur la place. Le village, à l'instar de la bipolarisation de la marka San Andrés en moitié haute et moitié basse, se divisait en deux parties. Lors des fêtes et pour effectuer les travaux, le haut du village était celui des ayllu de la moitié haute et le bas du village, celui des ayllu de la moitié basse. L'espace de la place, divisée en six parties - une pour chaque ayllu -, reproduisait fidèlement la disposition des ayllu sur le territoire de la marka selon les points cardinaux nord-sud et est-ouest.

14. Selon un système appelé " al partir », certains villageois (et de nombreux migrants) confient leurs troupeaux à des comunarios qui les font paitre avec leurs propres troupeaux sur leurs terres et sur celles que les villageois ont éventuellement conservées. Les villageois se doivent toutefois d'effectuer certains travaux qui nécessitent de la main d'œuvre (marquage des bêtes, tontes, etc.); les animaux nés dans l'année seront partagés avec le comumario qui les a fait paître.

15. Il s'agira de souligner les principales tendances chez ces trois groupes mais, bien évidemment, tous les membres de ces groupes n'adoptent pas la même attitude et la réalité de la marka San Andrés est autrement complexe. Certains commmarios n'ont plus aucun rapport avec le village et ses habitants ; parfois, les conflits et le séparatisme se situent au sein même d'un ayllu: les membres de certaines estancias, souhaitant créer un nouvel ayllu, rejoindre une marka voisine, etc., s'opposent dès lors aux membres d'autres estancias. Tous les vecinos ne reviennent plus au village : certains se sont établis dans les basses terres, dans la ville de Santa Cruz, d'autres sont partis vivre à l'étranger, généralement aux États-Unis, etc. Quant aux nouveaux villageois, ils sont originaires d'estancias et d'ayllu différents. Quelques-uns se disent être des " originaires du village " (originarios) lorsque leur intégration au village remonte à une génération, soit ils y sont arrivés dès leur enfance, soit des collatéraux y vivaient avant eux. Ils se distinguent de ceux qu'ils appellent les nouveaux venus (agregados) qui ont encore leurs parents ou collatéraux dans leur estancia d'origine, laquelle jouxte très souvent le village. Il s'agit de comunarios possédant peu de têtes de bétail mais une famille nombreuse qui s'occupe des bêtes, ce qui les laisse libres d'aller travailler ailleurs, de femmes demeurées seules avec leurs enfants et dont la famille ne peut pas pourvoir aux besoins et, de façon générale, de personnes en situation précaire dans leur estancia. Ces agregados retournent dans leur estancia une à deux fois par semaine pour surveiller les troupeaux familiaux. L'attitude des nouveaux villageois face aux comunarios est à multiples facettes selon les liens plus ou moins forts conservés avec leur estancia d'origine. J'ai déjà montré, par ailleurs, comment ce groupe - les nouveaux villageois - peinait à élaborer le ciment d'une identité collective et était déchiré par des conflits internes (Bouté 1998).

16. Pour les vecinos et les commmarios, j'ai repris les termes par lesquels ils s'auto-désignent. Les habitants du village utilisent pour s'auto-désigner une pluralité de termes (nosotros los del pueblo, los vecinos, la gente de vestido) variant selon leurs interlocuteurs. Les vecinos et les comunarios emploient également, pour désigner les nouveaux villageois, des termes variables selon les contextes : los indios, la gente de pollera, la cholada, pour les premiers; los del pueblo, el pueblo, los vecinos, pour les seconds. Pour ma part, j'ai retenu l'expression « nouveaux villageois » afin de qualifier ce groupe.

17. Le qualificatif « villageois » aurait prêté à confusion dans cet article puisque l'un des groupes est désigné par le terme " nouveaux villageois ». J'ai donc eu recours à une francisation de l'épithète espagnol « vecinal(e) » pour préciser les traits propres aux vecinos.

18. Cette aspiration à la modernité des vecinos prend pour le moment la forme de projets aussi somptueux qu'irréalisables.

19. Comme le note Albó (1977), s'enorgueillir de son identité «indienne » reste le fait d'une nouvelle élite; ces représentations ne correspondent pas toutefois à une réalité vécue, elles restent au niveau du discours.

20. Voir à ce propos Albó y Preiswerk (1986) et Abercrombie (1992).

21. Les mouvements indigénistes prennent forme à la fin du $\mathrm{xIX}^{\mathrm{e}}$ et au début du $\mathrm{xx}^{\mathrm{e}}$ siècle en Amérique latine. Manifestation d'une réflexion créole et métisse sur l'Indien, souvent fondés sur une représentation magnifiée du passé, les mouvements indigénistes prônaient l'intégration des populations indigènes (dont ils dénonçaient la condition) tout en tâchant d'asseoir une identité nationale sur la base de l'indianité. Ces mouvements furent rejetés par les courants indianistes qui se développèrent à partir des années 1970, l'indianisme se voulant l'expression de revendications spécifiquement indi- 
gènes et s'opposant à l'intégration aux États-nations occidentalisés pour promouvoir des nationalités indiennes (Favre 1996).

22. Ströbele-Gregor (1994, pp. 113, 115) note que le mouvement katariste - nommé ainsi en mémoire du leader aymara Tupac Katari du mouvement de révolte anticoloniale de 1781 - s'appuie sur une glorification de l'empire inca comme symbole de la résistance des populations andines à l'hégémonie coloniale. En 1984, l'un des points du programme du CSUTCB (Confederación sindical única de trabajadores y campesinos de Bolivia) était notamment la reconstruction d'un empire inca modernisé étendant ses frontières au-delà de la Bolivie.

23. Films, diffusés dans les écoles de Jesús de Machaqa et des marka voisines, proclamant notamment la renaissance d'une " grande nation andine ».

24. D'après Monast (1972, p. 205), étymologiquement le pasante est le porte-étendard - il fait passer la banderole du saint à travers le village - avec les charges que cet honneur implique.

25. Littéralement « le gage d'affection »; il s'agit en l'occurrence des offrandes à la divinité.

26. Les comumarios, d'ailleurs, finissent par intérioriser en partie ce discours, au point de se sentir moins « andins » que les vecinos.

27. Voir sur ce sujet l'article de Galinier et Molinié (1998).

28. Molinié propose de voir ce transfert de l'Indien dans un être désincarné comme une façon de l'expulser de la réalité. L'Indien, débarrassé de l'aura négative qui lui était accolée jusque-là, peut devenir sujet d'identification pour les Métis et les Créoles qui, de cette façon, peuvent se sentir proches des Incas et s'inventer une autochtonie sur un territoire qui devient leur (séminaire « Figures nationales de l'Indien ", Nanterre, département d'ethnologie, 1996-1997).

29. On reprend ici la définition donnée par Lenclud $(1994$, p. 33) à propos de la traditionnalité envisagée "comme le produit d'une construction hic et muc, "traditionnant" des discours et des événements quelle que puisse être la relation entretenue par ces discours et par ces événements avec le passé $"$.

30. Le terme de « residentes " (résidents) désigne l'ensemble des migrants, qu'ils soient vecinos ou commarios. Les residentes se reconnaissent entre eux selon leur lieu d'origine. Cette différenciation apparaît à plusieurs niveaux : des residentes ont un sentiment d'appartenance commune à la même région ; puis, ils distinguent entre eux ceux qui sont originaires de la même marka, du même ayllu, enfin de la même estancia. Les residentes venant des ayllu et ceux venant du village forment ainsi deux groupes distincts qui s'organisent en tant que tels dans la ville (le Centró Acción est le lieu de réunion des anciens vecinos à $\mathrm{La} \mathrm{Paz}$, tandis que les nouveaux villageois et les comumarios tendent plutôt à se regrouper selon leur communauté d'origine dans des centres d'entraide à Villa Fatima, quartier d'El Alto - la banlieue de La Paz où vit la majorité des migrants aymaras).

31. L'ensemble des charges comportait, avant 1953, plusieurs étapes où alternaient les charges religieuses dédiées aux saints patrons catholiques et les charges politiques traditionnelles (incluant les cultes aux divinités autochtones); les plus importantes étaient celles de jilakata pour l'ayllu et de cacique pour l'estancia. Actuellement, les paysans ont repris certaines caractéristiques de l'ancien système des charges (hiérarchie, rotation, fonction intégrative, etc.), mais les charges politiques semblent avoir perdu certaines fonctions rituelles autrefois dévolues aux jilakata. Ces charges politiques ou civiles durent un an et sont de deux types : les charges syndicales (subcentral, secrétaire général) et les charges spécifiques au village (maire, juge, agent cantonal, président de la Junta des vecinos, etc.).

32. Son rôle d'organisateur ne concerne cependant pas le déroulement des différentes étapes de la fète qui sont pratiquement toujours les mêmes.

33. Lors du baptême, de la première coupe de cheveux ou du mariage d'un enfant, le père et le parrain deviennent compadres. Les partenaires sont tenus de s'entraider à tous les niveaux; une forte pression sociale les contraint à répondre de leur mieux à cette exigence. Comme le note Molinié (1974, p. 1364), "le compadrazgo relève de formes de production anciennes dont la réciprocité était le fondement, tout en témoignant d'une manière indigène de vivre et dissimuler les rapports inégaux ».

34. La location des costumes, indispensables pour la participation à une comparsa, est onéreuse et se fait en ville. Le coût occasionné par la location du costume, le prix des transports et le temps pour se 
rendre à et revenir de La Paz (deux jours au moins) découragent généralement les rares comunarios invités par les vecinos.

35. Même si, comme le note Wachtel (1990, p. 133), il est difficile de faire la part de la spontanéité et celle de la pression du groupe.

36. Lors de ces fêtes, il n'y a généralement qu'un seul pasante, peu de danseurs et la fanfare est composée de cinq musiciens, tout au plus, recrutés parmi les comumarios des environs.

37. Sur l'introduction et la diffusion des Églises évangéliques en Bolivie, voir Rivière (1997).

38. Par exemple, lors des semailles, les nouveaux villageois et les comumarios catholiques font des offirandes à la Pachamama.

39. Le 16 juillet, tous les ayllu célébraient la Sainte Carmen dans le village. Cette fête était couramment appelée " la fête des ayllu » et les vecinos n'y participaient pas.

40. Autrefois, le curé (et plus généralement les Blancs et Métis) était considéré comme le kharikkari (ou lik'ichiri) c'est-à-dire un homme blanc qui, n'ayant de cesse que de sucer la graisse humaine, attaquait les Indiens. Selon la croyance des comunarios, le curé prélevait cette graisse pour la confection des cierges de l'Église. De nos jours, le kharikkari n'est plus exclusivement le curé, et les comunarios désignent comme tel de nombreux habitants du village : certains nouveaux villageois ayant la peau claire, l'infirmier prélevant la graisse sous couvert de vaccinations, des commnarios de Jésus de Machaqa employés comme techniciens par une ONG locale, etc. Le village apparait, selon leurs dires, comme un endroit dangereux qui menace leur intégrité physique.

41. Il m'a été difficile d'obtenir des renseignements. Certains nouveaux villageois racontent que des comunarios ont mené un véritable charivari durant l'oflice; pour d'autres, les comunarios auraient publiquement reproché au Père d'être du côté des vecinos et contre les habitants des communautés, etc. Quant au Père Pepe, son assistante m'ayant soupçonnée d'être une missionnaire pentecôtiste, je n'ai pu l'approcher.

42. Comme le souligne Rivière (1997, p. 97), « le groupe religieux est susceptible de fournir un espace, un support psychosocial et un cadre de référence à des individus qui refusent d'être enfermés dans une catégorie vécue négativement et qui voient dans les mécanismes sociaux traditionnels des facteurs de ségrégation et de marginalisation ".

43. Il est intéressant de noter que les deux grands courants concurrents, catholique et protestant, sont également présents à travers deux ONG, implantées chacune dans l'un des deux territoires voisins de San Andrés, Jesús de Machaqa et Santiago de Machaqa.

\section{BIBLIOGRAPHIE}

APTER D.

1965 The Politics of modernization, University of Chicago Press, London, Chicago.

ABERCrombie Thomas

1990 "Ethnogenèse et domination coloniale », Journal de la Société des Américanistes, 76, pp. 95-104.

1992 "To be Indian, to be Bolivian : "ethnic" and "national" discourses of identity ", in G. Urban and J. Sherzer, eds, Nations-States and Indians in Latin America, University of Texas Press, Austin, pp. 95-130.

Albó Javier

1977 Khitipxtansa. ¿Quiénes somos? Identidad localista, étnica y clasista en los Aymaras de hoy, CIPCA, Cuadernos de Investigación, La Paz. 
Albó Javier y Matias PreIswerk

1986 Los señores del Gran Poder, Centro de Teologia Popular, Alenkar, La Paz.

BAssoli Sergio

1988 Diagnóstico Cantón San Andrés de Machaqa. Estudio socioeconómico, Proyecto CIPCA-Sattawi, La Paz.

BourRICAUD François

1962 Changements à Puno, Institut des hautes études de l'Amérique Latine, Paris.

Bouté Vanina

1998 Identités en formation dans un village de l'Altiplano bolivien, mémoire de maîtrise, Université Paris X-Nanterre.

BouYSSE-CASSAGNe Thérèse

1978 "L'organisation de l'espace aymara », Annales, Anthropologie historique des sociétés andines, 5-6, pp. 1057-1080.

Choque Roberto

$1988 \quad$ Historia de Machaqa, Proyecto CIPCA-Sattawi, La Paz.

Claudel Cécile

2000 Se faire "vecino" à Mocomoco. Identités sociales et représentations de l'espace de la petite bourgeoisie rurale dans les Andes boliviennes, thèse de doctorat en ethnologie, EHESS, Paris.

FAVRE Henri

1996 L'indigénisme, Presses Universitaires de France, Paris, coll. «Que saisje ? » 3088.

Galinier Jacques et Antoinette Molinié

1998 "Le crépuscule des lieux. Mort et renaissance du musée d'Anthropologie », Gradhiva, 24, pp. 93-102.

Harris Olivia

1978 "De l'asymétrie au triangle : transformations symboliques », Annales, Anthropologie historique des sociétés andines, 5-6, pp. 1108-1125.

ISBELL Billy-Jean

1978 To defend ourselves. Ecology and ritual in an Andean village, University of Texas Press, Austin.

Lavaud Jean-Pierre

1998 «La Bolivie de la Réforme », Problèmes d’ Amérique Latine, 28, pp. 3-18.

\section{LeNClud Gérard}

1994 "Qu'est-ce que la tradition ? ", in Marcel Détienne, ed., Transcrire les mythologies, Albin Michel, Paris, pp. 25-44.

MoLINIÉ Antoinette

1974 "Débat 》, Annales, 6, pp. 1362-1365.

1982 La vallée sacrée des Andes, Société d'ethnographie, Paris.

1986 "Instruments symboliques aux frontières andines », Techniques et culture, 7, pp. 145-179. 
MONAST J.-E.

1972

Los Indios aimaraes; Evangelizados o solamente bautizados? Cuadernos Latinoamericanos, Carlos Lohlé, Buenos Aires.

PAREDES Manuel

1955 Tihuanacu y la Provincia Ingavi, Isla, La Paz.

PlatT Tristan

1978

«Symétries en miroir », Annales, Anthropologie historique des sociétés andines, 5-6, pp. 1081-1107.

Rıvıère Gilles

1983

«Quadripartition et idéologie dans les communautés aymaras de Carangas "), Bulletin de l'Institut français d'études andines, XII (3-4), pp. 41-62.

1997 "Bolivie : le pentecôtisme dans la société aymara des hauts plateaux ", Problèmes d'Amérique Latine, 24, pp. 81-102.

STRÖBELE-Gregor Juliana

1994 "From Indio to Mestizo... to Indio. New Indianist Movements in Bolivia ", Latin American Perspectives, 21 (2), pp. 106-123.

URTON Gary

1993

"Contesting the past in the Peruvian Andes ", in Antoinette Molinie et Aurore Monod Becquelin, eds, Mémoires de la tradition, Société d'ethnologie, Nanterre, pp. 107-144.

WACHTEL Nathan

1990 Le retour des ancêtres. Les indiens Urus de Bolivie, xx-xvf siècle. Essai d'histoire régressive, Gallimard, Paris. 